\title{
BMJ Open Protocol for a phase IV, open-label feasibility study investigating non- invasive markers of hepatic fibrosis in people living with HIV-1 and non- alcoholic fatty liver disease randomised to receiving optimised background therapy (OBT) plus maraviroc or OBT alone
}

Daniel Bradshaw (D) , ${ }^{1}$ Yvonne Gilleece, ${ }^{1}$ Sumita Verma, ${ }^{2}$ Iga Abramowicz, ${ }^{2}$ Stephen Bremner, ${ }^{2}$ Nicky Perry ${ }^{2}$

To cite: Bradshaw D, Gilleece Y, Verma S, et al. Protocol for a phase IV, open-label feasibility study investigating noninvasive markers of hepatic fibrosis in people living with HIV-1 and non-alcoholic fatty liver disease randomised to receiving optimised background therapy (OBT) plus maraviroc or OBT alone. BMJ Open 2020;10:e035596. doi:10.1136/ bmjopen-2019-035596

- Prepublication history for this paper is available online. To view these files, please visit the journal online (http://dx.doi. org/10.1136/bmjopen-2019035596).

Received 07 November 2019 Revised 22 April 2020 Accepted 01 May 2020

Check for updates

(c) Author(s) (or their employer(s)) 2020. Re-use permitted under CC BY-NC. No commercial re-use. See rights and permissions. Published by BMJ.

For numbered affiliations see end of article.

Correspondence to Dr Daniel Bradshaw; daniel.bradshaw2@nhs.net

\section{ABSTRACT}

Introduction At least $30 \%$ of people living with HIV (PLWH) infection have non-alcoholic fatty liver disease (NAFLD), which has now become a leading cause of hepatic fibrosis and cirrhosis. Management is based largely on lifestyle modifications, which are difficult to achieve, and therapeutic options are urgently needed. Maraviroc (MVC), through antagonism of CCR5 receptors, may reduce hepatic fibrosis progression and could be an effective treatment for NAFLD. However, dosing is usually two times per day, unlike most currently recommended antiretroviral therapies. This study will investigate the feasibility and acceptability of addition of MVC to combination antiretroviral therapy in PLWH and NAFLD as a treatment for NAFLD.

Methods and analysis This is a phase IV, randomised, open-label, non-invasive feasibility study. Sixty individuals with well-controlled HIV-1 and NAFLD will be recruited from UK HIV clinics and randomised 1:1 to receive either optimised background therapy (OBT) plus MVC or OBT alone. Follow-up will be every 24 weeks for 96 weeks. The primary outcome measures will include recruitment and retention rates, adverse events and adherence. Secondary outcomes will include changes in markers of hepatic fibrosis, including the Enhanced Liver Fibrosis score, median liver stiffness measurement and controlled attenuation parameter scores on Fibroscan, and quality of life assessments. Analyses will be performed according to intention-to-treat principles. For secondary outcomes, estimated differences and 95\% Cls between the groups using a t-method will be presented for continuous variables and as exact $95 \%$ binomial $\mathrm{Cls}$ for categorical variables.

Ethics and dissemination Ethical approval was obtained through the London Dulwich UK Research Ethics Committee (reference 17/L0/2093). Results will be

\section{Strengths and limitations of this study}

- Investigating a novel treatment for non-alcoholic fatty liver disease in people living with HIV (PLWH).

- Simple study design ensuring ease of understanding for potential recruits.

- Non-invasive approach likely to increase acceptability to participants.

- Minimal difference in frequency of follow-up from standard of care for PLWH.

- The gold standard for assessment of liver disease is biopsy; hence, non-invasive markers may underquantify or overquantify the degree of steatosis or fibrosis. However, a requirement for histology is likely to deter many potential recruits, particularly those unlikely to have advanced disease.

disseminated both through community groups and peerreviewed scientific literature.

Trial registration number SRCTN31461655. EudraCT number 2017-004141-24; Pre-results.

\section{INTRODUCTION}

Recent cohorts, and a systematic review, have identified a prevalence of non-alcoholic fatty liver disease (NAFLD) in people living with HIV (PLWH) of between $30 \%$ and $50 \% .{ }^{12}$ Risk factors include those associated with the metabolic syndrome, in particular, high body mass index (BMI), type II diabetes mellitus and dyslipidaemia, as well as genetic polymorphisms. Additional HIV-related risk factors include immunoactivation, gut microbiome dysregulation and antiretroviral 
therapy toxicity. ${ }^{34}$ Of those with NAFLD, $20 \%-40 \%$ will develop steatohepatitis, which may progress to fibrosis and cirrhosis. ${ }^{24}$ Thus, NAFLD has now become a leading cause of liver disease in PLWH.

Several management approaches are recommended for NAFLD. These include addressing underlying lifestyle factors, including reducing weight by $\sim 10 \%$, increasing exercise and optimising glycaemic control. ${ }^{5}$ Pharmacological interventions include vitamin $\mathrm{E}$ and pioglitazone. ${ }^{5}$ However, there are few data to inform optimal interventions, particularly in PLWH, which has led to increasing interest in novel approaches.

The chemokine CCL5/RANTES, the ligand for CCR5, plays a key role in hepatic inflammation and fibrosis. CCR5 mediates intrahepatic immune cell interactions which promote activation and migration of Kupffer cells and hepatic stellate cells; these in turn promote inflammation and hepatic fibrosis. ${ }^{6}$ Antagonism of this pathway could therefore reduce fibrosis progression. ${ }^{7-9}$

Maraviroc (MVC) is licensed for the treatment of HIV-1 infection in both treatment-naïve and treatmentexperienced individuals, where the infecting strain is R5 tropic, as part of combination antiretroviral therapy (cART). ${ }^{10}{ }^{11}$ MVC inhibits the binding of HIV-1 gp120 to the CCR5 coreceptor, thereby preventing virus entry into the cell. The property of MVC antagonising CCL5-CCR5mediated interactions has led to interest in its potential anti-inflammatory benefits, in addition to its anti-HIV activity, particularly in the liver and brain. ${ }^{12-14}$ Furthermore, there are over 10 years of data relating to the safety of MVC in humans, and the drug is well tolerated, including in individuals with chronic liver disease caused by HIV/HCV or HIV/HBV coinfection. ${ }^{15}$

In vitro, MVC reduces the release of proinflammatory cytokines implicated in fibrosis from immortalised human hepatic stellate cells, with reduced expression of extracellular matrix proteins. ${ }^{16} \mathrm{MVC}$ also reduces rates of hepatic fibrosis progression in mice. ${ }^{17}$

Furthermore, in an analysis of HIV/HCV-coinfected individuals receiving MVC-containing cART, MVC reduced hepatic fibrosis progression over 18 months, indicated through the aspartate aminotransferase (AST):platelet ratio index, with one of three individuals showing fibrosis regression. ${ }^{13}$ Transient elastography studies also demonstrated a benefit of MVC on liver stiffness measurements in HIV/HCV-coinfected individuals receiving MVC. ${ }^{18}$

Finally, in a phase two study, cART-naïve PLWH were randomised to receiving efavirenz or the novel antiretroviral, cenicriviroc (CVC), an antagonist of CCR2 and CCR5, both in combination with tenofovir disoproxil fumarate-emtricitabine. Those in the CVC arm experienced a decrease in the monocyte activation marker sCD14. ${ }^{19}$ Furthermore, in a phase two study of CVC in HIV-uninfected individuals with non-alcoholic steatohepatitis (NASH), twice as many individuals receiving CVC versus placebo achieved an improvement in biopsyassigned fibrosis . ${ }^{20}$

\section{Box 1 Primary outcome measures}

- Proportion of eligible individuals approached who are successfully recruited.

- Monthly participant recruitment rate.

- Participant retention in the study at 48 and 96 weeks.

- Proportion of participants for whom there are missing data at 48 and 96 weeks.

- Proportion of participants reporting adverse events at 48 and 96 weeks.

- Level of self-reported adherence to the study drug at 48 and 96 weeks in those allocated to the maraviroc group.

Following these observations, it is plausible that CCR5receptor antagonism by MVC may reduce hepatic fibrosis in PLWH and NAFLD. However, no study has yet investigated this.

\section{STUDY RATIONALE}

In vitro data for MVC and clinical trial outcomes for CVC suggest CCR5 antagonism may reduce hepatic fibrosis. MVC is licensed for HIV-1 treatment as part of cART and may be an effective treatment for NAFLD in PLWH. However, recommended dosing is two times per day, which could be associated with reduced acceptability to PLWH and NAFLD. There is therefore a need to investigate the feasibility and acceptability of the addition of MVC to antiretroviral therapy in PLWH and NAFLD as a possible therapeutic option for NAFLD.

\section{OUTCOME MEASURES}

Primary and secondary outcome measures are shown in boxes 1 and 2.

\section{Box 2 Secondary outcome measures}

Mean change in the Enhanced Liver Fibrosis score by 48 and 96 weeks.

- Mean change in Fibroscan median liver stiffness measurement by 48 and 96 weeks.

- Mean change in the Fibroscan controlled attenuation parameter score by 48 and 96 weeks.

- Change in the $\%$ with a CT liver:spleen attenuation ratio of $<1.0$ by 96 weeks.

- Mean change in blood-derived biochemistry by 48 and 96 weeks: fasting high-density lipoprotein (HDL):cholesterol ratio, lowdensity lipoprotein, $\mathrm{HDL}$, triglyceride, glucose, $\mathrm{Hb} 1 \mathrm{Ac}$ and alanine aminotransferase.

- Mean change in clinical signs of the metabolic syndrome by 48 and 96 weeks: body mass index, waist circumference and weight.

- Mean change in HIV parameters: CD4 cell count and per cent with undetectable HIV viral load.

- Differences in the quality of life of participants by 48 and 96 weeks as assessed by responses to the chronic liver disease questionnaire (CLDQ) for non-alcoholic fatty liver disease, and the 36-Item Short Form Survey and Work Productivity and Activity Impairment: Specific Health Problem (WPAI:SHP) questionnaires. 


\section{Rationale for primary outcome measures}

Primary outcome measures will assess the feasibility and acceptability of addition of MVC to effective cART in a cohort of PLWH as a possible therapy for NAFLD. Acceptability will be considered through evaluation of participant recruitment rate, retention rate and adherence. Should feasibility and acceptability be confirmed, this would support the establishment of a larger, randomised, double-blind, placebo-controlled study to assess the efficacy of the intervention.

\section{Rationale for secondary outcome measures}

Measures of the efficacy of MVC on a combination of non-invasive markers of hepatic inflammation and fibrosis will be identified, including the ELF score and median liver stiffness measurement and controlled attenuation parameter (CAP) scores on Fibroscan. Invasive procedures have been avoided, as liver biopsy would deter potential participants, particularly those without advanced liver disease.

\section{TRIAL DESIGN}

This is a phase IV, open-label, randomised, dual-arm feasibility study. Randomisation will be stratified according to

1. Current exposure or past history of $\geq 6$ months' exposure to protease inhibitor (PI)-containing antiretroviral therapy versus no current exposure and $<6$ months past exposure to PI-containing therapy.

2. BMI of $\geq 25$ versus $<25$.

3. Current exposure to a lipid-lowering agent (eg3hydroxy-3-methyl-glutaryl-coenzyme A (HMG CoA) reductase inhibitors, eg, statins; cholesterol absorption inhibitors, eg, ezetimibe; bile acid-binding drugs, eg, cholestyramine; fibrates; and omega 3 fatty acids).

4. Diabetes mellitus (DM) status ( 1 or 2 vs no DM)

Stratification will be undertaken to balance the treatment groups on important prognostic factors (ie, to prevent confounding), given that high BMI, $\mathrm{DM}^{2}$ and concurrent administration of $\mathrm{PIs}^{1}$ have been associated with faster hepatic fibrosis progression. Statin use has conversely been associated with relative protection from fibrosis. ${ }^{21}$

Blinding will not be used for this feasibility study, although results may inform the design of a subsequent larger, blinded placebo-controlled randomised controlled trial investigating efficacy of the intervention. However, it is unlikely that there will be behavioural differences in the MVC versus non-MVC group, which would change risk of fibrosis progression (such as dietary modifications, increase in exercise or reduction in alcohol consumption). Therefore, the use of placebo is not considered essential.

\section{ELIGIBILITY CRITERIA}

Inclusion and exclusion criteria are shown in boxes 3 and 4 .

\section{Box 3 Inclusion criteria}

- Aged 18 years and older.

- HIV-1 infected with durably suppressed HIV viral load (VL) ( $<50$ copies/mL for $\geq 6$ months).

- Note: one HIV VL blip (VL 50-200 copies/mL) is allowed in the 6 months prior to screen.

- Has evidence of NAFLD on hepatic imaging (USS, CT or MRI) or on biopsy either at screen or in the 6 months prior to screen.

- Provides written, informed consent to participate.

- Is willing to comply with the protocol requirements.

- If female and of childbearing potential, is using effective birth control methods (as agreed by the investigator) and willing to continue practising these birth control measures during the trial and for at least 30 days after the end of the trial.

Note: Women who have been postmenopausal for at least 2 years, women with a total hysterectomy and women who have had a tubal ligation are considered of non-childbearing potential.

- If male and sexually active with female partners of childbearing potential, is using effective barrier contraception and willing to continue using this during the trial and for at least 30 days after the end of the trial.

\section{Box 4 Exclusion criteria}

- Severe cardiovascular disease, including known angina or history of myocardial infarction.

- History of postural hypotension, defined as a reduction in the systolic blood pressure of $\geq 20 \mathrm{~mm} \mathrm{Hg}$ after standing for at least $1 \mathrm{~min}$.

- Individuals previously exposed to maraviroc (MVC).

- HIV viral load (VL) detectable (>50 copies $/ \mathrm{mL}$ ).

Note: one blip (VL 50-200 copies/mL) within 6 months prior to screen is allowed.

- Current HCV or HBV (HBcAb-positive,HBsAg-negative is permitted; HCV antibody positive with HCV RNA or HCV antigen negative for $\geq 6$ months following treatment or spontaneous clearance is permitted).

- Other chronic liver disease, including but not exclusively, cirrhosis, ${ }^{*}$ alcohol-related liver disease, $\uparrow$ autoimmune hepatitis, primary biliary cirrhosis, primary sclerosing cholangitis, haemochromatosis, Wilson's disease, alpha-1 antitrypsin deficiency and drug-induced non-cirrhotic portal hypertension, as deemed by a hepatologist.

- Alanine aminotransferase or aspartate aminotransferase of $>205$ IU/L.

- Severe renal insufficiency (creatinine clearance of $<30 \mathrm{~mL} / \mathrm{min}$ ).

HIV-2 infection.

- Known allergy or intolerance to MVC or its constituents, including hypersensitivity to peanuts or soya.

If female, pregnancy or breast feeding.

- Individuals currently taking medications or herbal agents that are contraindicated with MVC, including St John's wort.

*Confirmed on biopsy, appearance on imaging (nodular liver with features of portal hypertension, such as splenomegaly of $>13 \mathrm{~cm}$ and/or varices) or liver stiffness measurement score ( $>13 \mathrm{kPa})$.

tIncludes liver disease in the presence of excess alcohol intake as defined according to European Association for the Study of the Liver guidelines 2016 (ie, $>20 \mathrm{~g} /$ day or $>17$ units/week for women and $>30 \mathrm{~g} /$ day or $>26$ units/week for men). 
Table 1 Summary of trial procedures

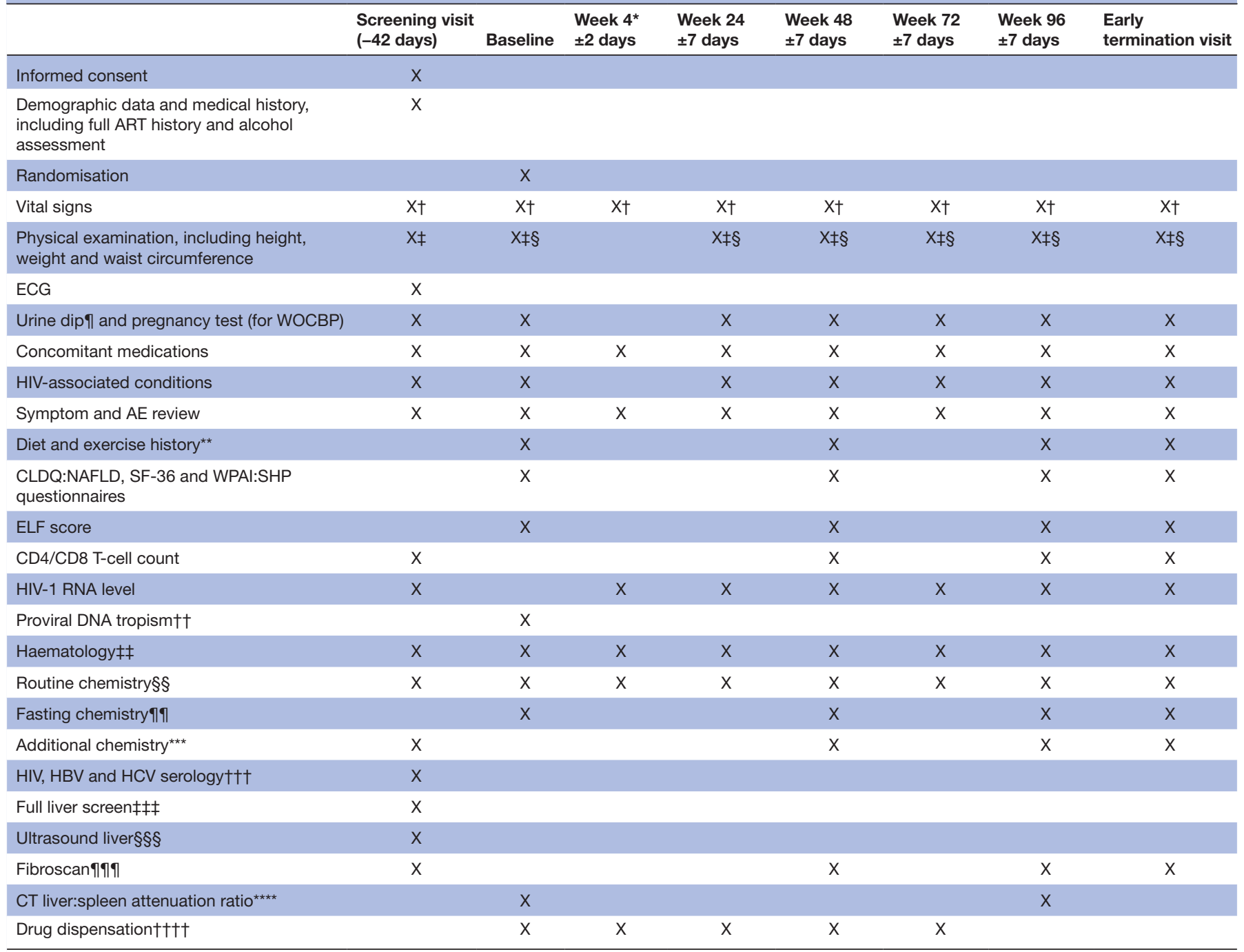

*Week 4 visit only for individuals receiving MVC. Blood is unfasted.

tHeart rate, respiratory rate, temperature, BP, lying and standing BP (postural BP at screening only and to be repeated if history indicates).

$\ddagger$ Height only at screening; weight and waist circumference at every visit except week 4.

$\S$ Symptom directed physical examination only.

IPoint of care urine dip for haematuria, proteinuria, glycosuria, leucocytes and nitrites.

${ }^{* *}$ Dietary history will be daily intake of olive oil, fruit, vegetables or salad, legumes, fish, wine, meat, white bread, rice and whole-grain bread. ${ }^{29}$ Exercise history will be the number of times per week exercise is undertaken, number of minutes of exercise per episode and type of exercise.

t†lf no result within the preceding 24 weeks.

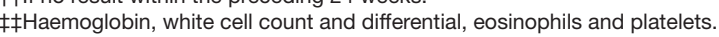

$\S \S S o d i u m$, potassium, chloride, creatinine, urea, alanine aminotransferase, aspartate aminotransferase, bilirubin, alkaline phosphatase, gamma glutamyltransferase, albumin,

phosphate, creatine kinase, glucose (screening, weeks 4, 24 and 72), lipids (total cholesterol, HDL, LDL and TGs) (weeks 24 and 72 only).

IIIFasting glucose and fasting lipids (total cholesterol, HDL, LDL, TGs).

***Haemoglobin A1c.

†††HCV antibody, HCV RNA or HCV antigen, HBsAg; if no prior record of result: HBcAb. HIV antibody-antigen only if no previous documented result.

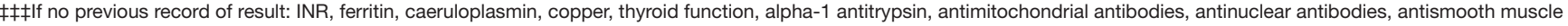
antibody, antiliver/kidney/microsomal antibodies-1 and coeliac serology.

$\S \S \S$ If no previous imaging (ultrasound, CT or MRI) result confirming fatty liver in the preceding 24 weeks.

ๆๆ IIncludes both median stiffness and controlled attenuation parameter scores, to be performed within 7 days of the study visit.

${ }_{\star \star \star \star *}$ Optional. To be performed within 7 days of the study visit. Preference is for the 7 days prior to baseline.

††††Only for individuals assigned to the MVC group.

$\mathrm{AE}$, adverse event; ART, antiretroviral therapy; BP, blood pressure; CK, creatine kinase; CLDQ:NAFLD, chronic liver disease questionnaire for non-alcoholic fatty liver disease; ELF, enhanced liver fibrosis; $\mathrm{HBsAb}$, hepatitis B core antibody; $\mathrm{HBsAg}$, hepatitis B surface antigen; HDL, high-density lipoprotein; INR, international normalised ratio; LDL, low-density lipoprotein; MVC, maraviroc; SF-36, 36-Item Short Form Survey; TG, triglycerides; US, ultrasound; WOCBP, women of childbearing potential; WPAl:SHP, Work Productivity and Activity Impairment: Specific Health Problem Questionnaire.

\section{TRIAL PROCEDURES}

The schedule of assessments is summarised in table 1.

\section{RECRUITMENT}

Sixty participants will be recruited in seven UK National Health Service (NHS) HIV clinics. Potentially eligible individuals with evidence of hepatic steatosis on imaging or biopsy performed as part of routine clinical care will be identified by clinical staff, either from the direct care 
team or by research clinicians working in the same HIV team. It is envisaged that ultrasound will be used to identify possible participants as this is the recommended first-line diagnostic imaging procedure for NAFLD, ${ }^{22}$ although some cases, particularly individuals with mild hepatic steatosis, may be missed. It is also emphasised that this trial will recruit only PLWH with NAFLD who do not have cirrhosis. People with cirrhosis have been excluded in order that the study population is not too heterogeneous. However, people with cirrhosis would be included in a larger randomised controlled trial.

The following approach will be used for identification of participants: (1) review of a database of patients in the HIV department with NAFLD, (2) preidentification of patients due to attend a routine prearranged follow-up appointment in the HIV or hepatology service and (3) review of medical notes during routine clinical follow-up. Individuals will be contacted either in person in the clinic or by telephone. Anonymised information on individuals who are contacted but are not randomised will include age, gender, ethnicity, the reason for lack of eligibility for participation or if they are eligible but declined.

\section{STUDY VISITS}

Study visits and assessments will take place according to the schedule in table 1.

\section{Screening visit}

Potentially eligible participants will be invited to attend for an appointment, having been provided with a participant information sheet. Adequate time (at least 24 hours) will be allowed for questions and to consider the study before agreeing to participate. Written informed consent will be received by the investigator. Results of screening evaluations must be available within 42 days of randomisation and eligibility must be established.

\section{Randomisation scheme}

Subjects will be randomised 1:1 into the MVC and nonMVC groups at the baseline visit. In addition, there will be stratification according to the factors outlined above. The web-based Sealed Envelope system will be used to allocate individuals randomly to the MVC or non-MVC groups. The statistician will provide the randomisation list and the HEPatic fibrosis in people living with HIV-1 and nonalcoholic fatty liver disease randomised to receiving optimised background therapy (OBT) plus MARaviro $\underline{\text { or OBT }}$ (HEPMARC) Randomisation Guide will be followed by the study team.

\section{Withdrawal}

A participant is free to withdraw from the study at any time. In addition, the investigator may decide, for reasons of medical prudence, to withdraw a subject. If a subject discontinues study medication dosing, every attempt should be made to keep the subject in the study and continue to perform the required study-related procedures and follow-up procedures. If this is not possible or acceptable to the subject or investigator, the subject may be withdrawn from the study.

Study medication may also be discontinued in the following instances:

1. If the subject withdraws his/her consent.

2. If the investigator considers that it is in the best interest of the subject (eg intercurrent illness or unacceptable toxicity) for him/her to withdraw consent.

3 . The subject fails to comply with the protocol requirements or fails to cooperate with the investigator.

4. Pregnancy during the course of the study.

\section{TRIAL MEDICATION}

Name and description of the investigational medicinal product Maraviroc (Celsentri)

In the UK, MVC is a licensed drug indicated with other antiretroviral medications for treatment-experienced adults infected with only CCR5-tropic HIV-1. It is supplied as a film-coated tablet. The recommended dose is 150 , 300 or $600 \mathrm{mg}$ two times per day, depending on interactions with coadministered antiretroviral therapy and other medicinal products, according to the Summary of Product Characteristics (SPC). ${ }^{23}$

\section{Dosage schedules}

For those randomised to receiving MVC, dosing will be according to SPC recommendations. The total dose will be determined according to interactions with other medications. Modification of the dose of MVC will not be undertaken except where indicated due to potential drug-drug interactions (DDIs) with concomitant medications. Where a participant initiates a new medication or discontinues an existing medication, the investigator will confirm whether or not a DDI could occur and hence the need for any dose modification of MVC.

For women of childbearing potential, contraception needs to be used for the duration of the study and for 30 days after the end of the trial. This includes the following, according to the woman's preference and DDIs with concomitant medications:

- Intrauterine device.

- Hormonal-based contraception (pill, contraceptive injection, implant and intrauterine system).

- Double-barrier contraception (condom and occlusive cap, eg, diaphragm or cervical cap with spermicide).

- True abstinence.

\section{Assessment of compliance}

Compliance will be assessed through

1. Self-reporting of doses of MVC taken via a diary card.

2. Pill counting at each visit by a pharmacist and recording of the number of pills returned.

Participants will bring in all pill bottles at each study visit. The total number of investigational medicinal product (IMP) pills remaining at each visit will be counted and 
then returned to the participant to take until the bottle is finished.

The percentage of IMP compliance for each participant will be calculated. Where this figure is $<80 \%$, this will lead to a likely recommendation to discontinue IMP, although this will be at the discretion of the principal investigator. In all circumstances, every effort will be made to continue to follow-up the participant on the study. Of note, where a discrepancy exists between self-reported compliance and compliance identified via pill counting, any decision to discontinue IMP will rest with the study principal investigator.

\section{Toxicity management}

In the event of toxicity or intolerance to MVC in this study, subjects will be managed as in standard clinical practice. This may involve discontinuing MVC in some cases.

\section{ADVERSE EVENTS}

Adverse events (AEs) observed by the investigator or reported by the subject and any remedial action taken will be recorded in the subject's case report form (CRF) and should be verifiable in the subject's notes throughout the study. The nature of each event, time of onset after drug administration, duration and severity will be documented, together with the investigator's opinion of the causal relationship to the treatment (unrelated, unlikely, possible, probable and definite).

All subjects experiencing AEs, whether considered associated with the use of the study medication or not, must be monitored until the symptoms subside and any clinically relevant changes in laboratory values have returned to baseline or until there is a satisfactory explanation for the changes observed. Clinically significant changes in physical examination and blood safety profiles should also be recorded as AEs.

\section{Assessment of intensity}

Severity should be recorded and graded according to the AIDS Clinical Trial Group Grading Scale.

AEs, serious adverse events (SAEs), adverse reactions (ARs), serious ARs and suspected unexpected serious ARs may be directly observed and reported spontaneously by the subject or by questioning the subject at each study visit. These will be followed up until they are resolved or the subject's participation in the study ends (ie, until the final CRF is completed for that subject). In addition, all SAEs assessed by the investigator as possibly related to the investigational medication should continue to be followed up even after the subject's participation in the study is over.

Such events should be followed up until resolution or until no further change can reasonably be expected.

\section{Data safety and monitoring board (DSMB)}

A group of independent clinicians and a statistician comprise the DMSB. The DSMB will periodically review overall safety data according to its charter (available from Brighton Clinical Trials Unit), in addition to all other study parameters. It will determine patterns and trends of events, or identify safety issues, which would not be apparent on an individual case basis.

\section{POST-TRIAL CARE}

Post trial, all individuals will be provided with standard of care interventions indicated for the treatment of NAFLD in line with current UK National Institute for Health and Care Excellence (NICE) guidelines.

All participating individuals will be advised, as part of the informed consent process, that MVC would be discontinued post-trial. Where individual patients and/or their treating clinicians request the continuation of MVC post trial, this request will be considered on a case by case basis by the HIV multidisciplinary team at each site. Although the primary objective of the current study does not include efficacy of the drug, a substantial improvement in hepatic fibrosis markers in individual patients is likely to strengthen the case for continuation of drug, which will be made based on its need within the overall HIV treatment regimen.

In addition, any beneficial effect identified in the trial will inform the establishment of a larger study on the effect of MVC on NAFLD in PLWH. This, in turn, may lead to a change to national recommendations with the possibility that MVC could be prescribed to patients through existing NHS funding schemes.

\section{STATISTICS AND DATA ANALYSIS \\ Sample size calculation}

As this is a feasibility study, no formal sample size calculation has been conducted. Results may be used to estimate the variability of the treatment effect of MVC on the ELF score, which may inform the sample size calculation for a larger, randomised placebo-controlled trial.

In a previous biopsy study, a unit increase of 1 in the ELF score was associated with a 2.5-fold increased risk of a liver-related event (adjusted for age and stage of fibrosis), and therefore a unit increase of 1 is deemed to represent a clinically important entity. ${ }^{24}$ Other studies have confirmed the accuracy of the ELF score in the assessment of hepatic fibrosis in PLWH and in those with NAFLD. ${ }^{25-28}$ Assuming the SD of the ELF score is 1.12, with 20 patients in each group for the analysis, a difference in ELF of 1 point can be estimated with a 95\% CI from 0.6 to 1.4. Assuming an attrition rate of $33 \%$, a target of 30 individuals will be recruited per group. ${ }^{22}$

A t-method will be used to estimate the difference in ELF scores, together with 95\% CIs, between the two groups. $\mathrm{P}$ values will not be presented.

\section{Planned recruitment rate}

An estimated three to four individuals will be recruited per month over an 18-month period. This takes into account the following factors: 
- The estimated prevalence of NAFLD among HIVinfected cohorts is predicted to be $30 \%$. Although many individuals are undiagnosed, recruitment at seven sites with $>5000$ HIV-infected patients overall is expected to yield at least 200 who have been diagnosed with NAFLD on previous imaging as part of routine clinical care.

- All PLWH are expected to attend a minimum of two medical appointments per year. For a minimum of 5000 individuals, a monthly target of four to five is predicted to be feasible, allowing for one to two screen failures per month, leaving three to four to be recruited.

\section{Statistical analysis plan}

Summary of baseline data and flow of patients

Baseline comparability between the two randomised groups will be described by considering the variables below.

For categorical data, percentage will be presented. For continuous data, the mean or median will be presented for variables following a normal and non-normal distribution, respectively, in addition to the IQR.

- Age.

- Per cent male.

- Per cent of each ethnicity.

- Duration of HIV infection.

- Nadir CD4 count.

- Baseline CD4 count.

- Per cent with undetectable HIV viral load.

- Per cent receiving PI-based cART versus non-PI-based cART.

- Per cent receiving concomitant lipid-lowering therapy.

- BMI.

- Waist circumference.

- Weight.

- Blood pressure.

- Fasting glucose.

- HbAlc.

- Bilirubin.

- ALT.

- Fasting TG.

- Fasting LDL.

- Fasting HDL.

- Fasting total cholesterol.

- Fasting HDL:cholesterol ratio.

- Baseline ELF score.

- Baseline Fibroscan stiffness result.

- Baseline Fibroscan CAP score.

- \% with a CT liver:spleen attenuation ratio < 1.0.

- Baseline diet score.

See figure 1 for the Consolidated Standards of Reporting Trials flow diagram for this study.

\section{Feasibility outcome analyses}

All analyses will be performed on available cases according to intention-to-treat (ITT) principles. Therefore, all individuals randomised within the study (including those subsequently withdrawn) will be included in outcome analyses.
The following analyses will be presented:

1. Proportion of eligible individuals approached who are successfully recruited. It is envisaged that a recruitment rate of at least $50 \%$ will achieved. This takes into account the likelihood that a proportion of potential recruits will decline, for example, owing to the requirement for two times per day pill taking.

2. Monthly participant recruitment rate. This is expected to be at least two participants consistently per month, with an average of three to four.

3. Participant retention in the study at 48 and 96 weeks in the MVC and non-MVC assigned groups. This is expected to be at least $65 \%$ (cf $35 \%$ discontinuation in the Maraviroc versus Optimized Therapy in Viremic Antiretroviral Treatment-Experienced Patients (MOTIVATE) study ${ }^{10}$ ).

4. Proportion of participants for whom there are missing data at 48 and 96 weeks in the MVC and non-MVC assigned groups. This is expected to be less than $20 \%$ for all participants.

5. Proportion of participants reporting AEs at 48 and 96 weeks in the MVC and non-MVC assigned groups. This is expected to be less than $10 \%$ based on the SPC for MVC. ${ }^{23}$

6. Level of self-reported adherence to the study drug at 48 and 96 weeks in those allocated to the MVC group. This is expected to be greater than $90 \%$.

\section{Secondary outcome analysis}

The analysis of the secondary outcomes, shown in box 2 , will be presented as the estimated difference and 95\% CI between groups using a t-method for continuous variables and as exact $95 \%$ binomial CIs for categorical variables, from baseline to 48 and 96 weeks.

In the event of missing data, only available data will be included in the analyses.

\section{DATA HANDLING, CONFIDENTIALITY AND MONITORING}

An electronic data management system MACRO will be used in this study. All information will be recorded in source data and documentation that will be filed in patients' notes. Direct access to data will be granted to authorised representatives from the sponsor, host institution and the regulatory authorities when appropriate to permit trial-related monitoring, audits and inspections. A specific trial monitoring plan has been developed for the study (available from Brighton Clinical Trials Unit).

All investigators and trial site staff will comply with the requirements of the Data Protection Act 1998.

Personal information will be collected, kept secure and maintained. This will involve the creation of coded, depersonalised data, secure maintenance of the data and the linking code in separate locations using encrypted digital files and limiting access to the minimum number of individuals necessary for quality control, audit and analysis. The confidentiality of data will be preserved when the data are 


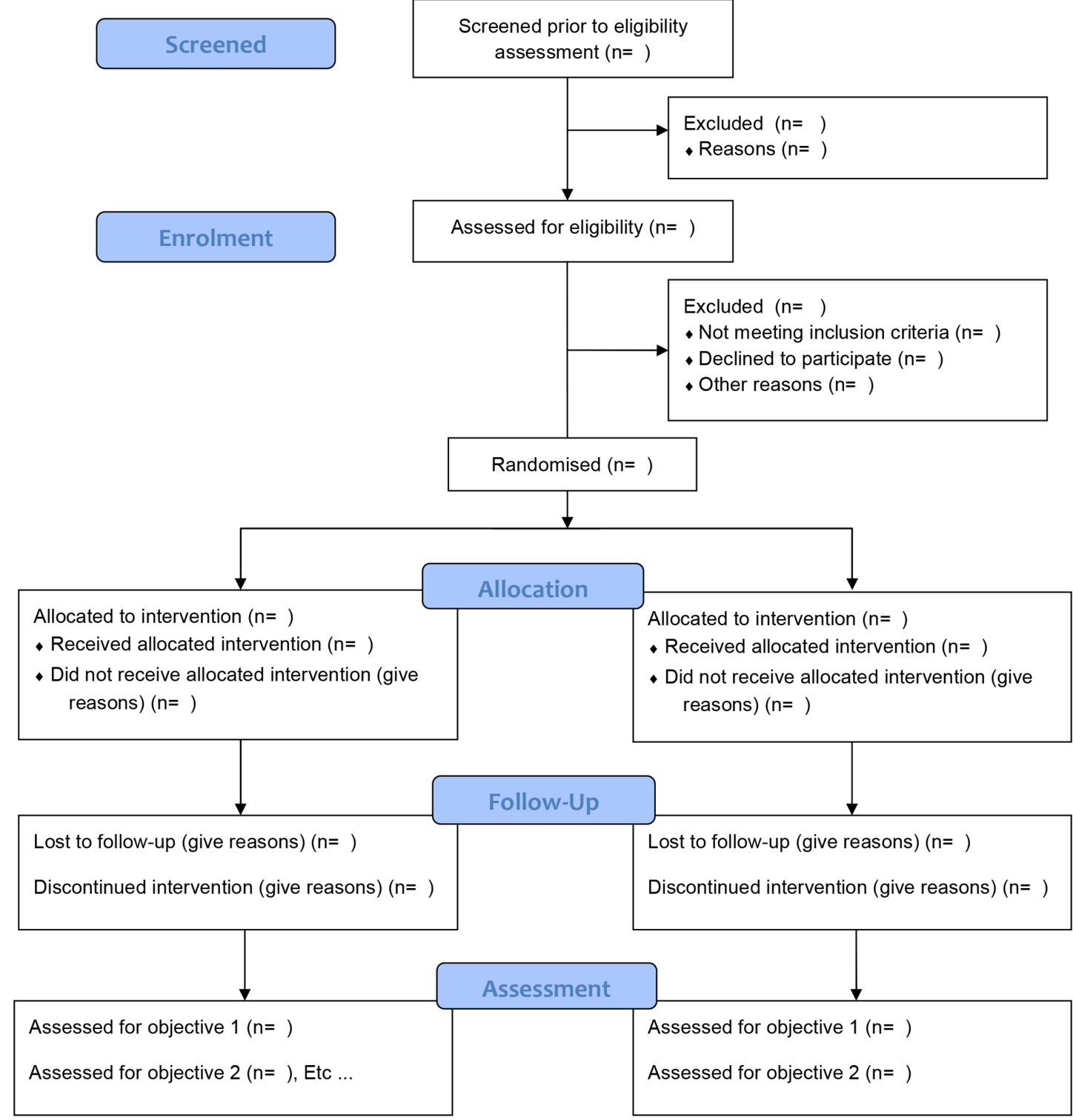

Figure 1 Consolidated Standards of Reporting Trials flow diagram for the HEPatic fibrosis in people living with HIV-1 and nonalcoholic fatty liver disease randomised to receiving optimised background therapy (OBT) plus MARaviroCor OBT (HEPMARC) study.

transmitted to sponsors and coinvestigators by using only pseudynomised codes rather than personal identifiable information. Data will be stored for 25 years.

\section{ETHICS AND DISSEMINATION}

This study has been approved by the Research Ethics Committee, London Dulwich, UK (REC reference 17/ LO/2093). Protocol amendments will be communicated to all relevant parties. Results of the study will be written up and submitted for publication in both HIV and hepatology journals, as well as disseminated through HIV community groups. Study data will be made available on reasonable request to the Brighton Clinical Trials Unit.

Author affiliations

${ }^{1}$ Brighton and Sussex University Hospitals NHS Trust, Brighton
${ }^{2}$ Brighton and Sussex Medical School, Brighton, UK

Contributors DB led on the study concept and writing the protocol. NP and IA assisted in writing and revising the protocol, including the safety assessments. YG and SV assisted in writing the clinical sections of the protocol. SB wrote the statistical section and analysis plan.

Funding This work was supported by Viiv Healthcare (reference number 207 967). Competing interests None declared.

Patient and public involvement statement Patients were involved in the study design and a patient representative sits on the Trial Steering Committee.

Patient consent for publication Not required.

Provenance and peer review Not commissioned; externally peer reviewed.

Open access This is an open access article distributed in accordance with the Creative Commons Attribution Non Commercial (CC BY-NC 4.0) license, which permits others to distribute, remix, adapt, build upon this work non-commercially, and license their derivative works on different terms, provided the original work is properly cited, appropriate credit is given, any changes made indicated, and the use is non-commercial. See: http://creativecommons.org/licenses/by-nc/4.0/. 
ORCID iD

Daniel Bradshaw http://orcid.org/0000-0001-7186-2482

\section{REFERENCES}

1 Vuille-Lessard Élise, Lebouché B, Lennox L, et al. Nonalcoholic fatty liver disease diagnosed by transient elastography with controlled attenuation parameter in unselected HIV monoinfected patients. AIDS 2016;30:2635-43.

2 Maurice JB, Patel A, Scott AJ, et al. Prevalence and risk factors of nonalcoholic fatty liver disease in HIV-monoinfection. AIDS 2017;31:1621-32.

3 Tafesh ZH, Verna EC. Managing nonalcoholic fatty liver disease in patients living with HIV. Curr Opin Infect Dis 2017;30:12-20.

4 Seth A, Sherman KE. Fatty liver disease in persons with HIV infection. Top Antivir Med 2019;27:75-82.

5 NICE. Non-alcoholic fatty liver disease (NAFLD): assessment and management, 2016. Available: https://www.nice.org.uk/guidance/ NG49/chapter/Recommendations\#lifestyle-modifications-for-nafld

6 Schwabe RF, Bataller R, Brenner DA. Human hepatic stellate cells express CCR5 and RANTES to induce proliferation and migration. Am J Physiol Gastrointest Liver Physiol 2003;285:G949-58.

7 Seki E, De Minicis S, Gwak G-Y, et al. Ccr1 and CCR5 promote hepatic fibrosis in mice. J Clin Invest 2009;119:1858-70.

8 Berres M-L, Koenen RR, Rueland A, et al. Antagonism of the chemokine CCL5 ameliorates experimental liver fibrosis in mice. J Clin Invest 2010;120:4129-40.

9 Barashi N, Weiss ID, Wald O, et al. Inflammation-Induced hepatocellular carcinoma is dependent on CCR5 in mice. Hepatology 2013;58:1021-30.

10 Gulick RM, Lalezari J, Goodrich J, et al. Maraviroc for previously treated patients with R5 HIV-1 infection. N Engl J Med 2008;359:1429-41.

11 Cooper DA, Heera J, Goodrich J, et al. Maraviroc versus efavirenz, both in combination with zidovudine-lamivudine, for the treatment of antiretroviral-naive subjects with CCR5-tropic HIV-1 infection. J Infect Dis 2010;201:803-13.

12 Martin-Blondel G, Brassat D, Bauer J, et al. CCR5 blockade for neuroinflammatory diseases--beyond control of HIV. Nat Rev Neurol 2016;12:95-105.

13 Gonzalez EO, Boix V, Deltoro MG, et al. The effects of maraviroc on liver fibrosis in HIV/HCV co-infected patients. J Int AIDS Soc 2014;17:19643.

14 Blanco J-R, Ochoa-Callejero L. Off-Label use of maraviroc in clinical practice. Expert Rev Anti Infect Ther 2016;14:5-8.

15 Rockstroh JK, Soriano V, Plonski F, et al. Hepatic safety in subjects with HIV-1 and hepatitis $\mathrm{C}$ and/or B virus: a randomized, doubleblind study of maraviroc versus placebo in combination with antiretroviral agents. HIV Clin Trials 2015;16:72-80.

16 Martini S, Perna A, Lucariello A. Effects of treatment with maraviroc - a CCR5 inhibitor on human hepatic stellate cells. Conference on
Retroviruses and Opportunistic Infections; 13-16 February, Seattle, 2017.

17 Pérez-Martínez L, Pérez-Matute P, Aguilera-Lizarraga J, et al. Maraviroc, a CCR5 antagonist, ameliorates the development of hepatic steatosis in a mouse model of non-alcoholic fatty liver disease (NAFLD). J Antimicrob Chemother 2014;69:1903-10.

18 Nasta P, Gatti F, Borghi F. (LS) change in HIV-hepatitis C (HCV) coinfected patients treated with CCR5 inhibitor based antiretrovira therapy. 51st Interscience Conference on Antimicrobial Agents and Chemotherapy; 17-20 September, Chicago, 2011.

19 Thompson M, Saag M, DeJesus E, et al. A 48-week randomized phase $2 \mathrm{~B}$ study evaluating cenicriviroc versus efavirenz in treatmentnaive HIV-infected adults with $\mathrm{C}-\mathrm{C}$ chemokine receptor type 5-tropic virus. AIDS 2016;30:869-78.

20 Friedman SL, Ratziu V, Harrison SA, et al. A randomized, placebocontrolled trial of cenicriviroc for treatment of nonalcoholic steatohepatitis with fibrosis. Hepatology 2018;67:1754-67.

21 Dongiovanni P, Petta S, Mannisto V, et al. Statin use and non-alcoholic steatohepatitis in at risk individuals. $J$ Hepatol 2015;63:705-12.

22 European Association for the Study of the Liver (EASL), European Association for the Study of Diabetes (EASD), European Association for the Study of Obesity (EASO). EASL-EASD-EASO clinical practice guidelines for the management of non-alcoholic fatty liver disease. $J$ Hepatol 2016;64:1388-402.

23 Viiv healthcare. Celsentri: summary of product characteristics. Available: https://www.medicines.org.uk/emc/product/6159/smpc [Accessed 18 May 2020].

24 Irvine KM, Wockner LF, Shanker M, et al. The enhanced liver fibrosis score is associated with clinical outcomes and disease progression in patients with chronic liver disease. Liver Int 2016;36:370-7.

25 Sherman KE, Abdel-Hameed EA, Ehman RL, et al. Validation and refinement of noninvasive methods to assess hepatic fibrosis: magnetic resonance elastography versus enhanced liver fibrosis index. Dig Dis Sci 2020;65:1252-7.

26 Inadomi C, Takahashi H, Ogawa Y, et al. Accuracy of the enhanced liver fibrosis test, and combination of the enhanced liver fibrosis and non-invasive tests for the diagnosis of advanced liver fibrosis in patients with non-alcoholic fatty liver disease. Hepatol Res 2020;50:690-692. doi:10.1111/hepr.13495. [Epub ahead of print: 23 Feb 2020].

27 Staufer K, Halilbasic E, Spindelboeck W, et al. Evaluation and comparison of six noninvasive tests for prediction of significant or advanced fibrosis in nonalcoholic fatty liver disease. United European Gastroenterol J 2019;7:1113-23.

28 Guillaume M, Moal V, Delabaudiere C, et al. Direct comparison of the specialised blood fibrosis tests FibroMeter ${ }^{\mathrm{V} G}$ and Enhanced Liver Fibrosis score in patients with non-alcoholic fatty liver disease from tertiary care centres. Aliment Pharmacol Ther 2019;50:1214-22.

29 Martínez-González MA, Fernández-Jarne E, Serrano-Martínez M, et al. Development of a short dietary intake questionnaire for the quantitative estimation of adherence to a cardioprotective Mediterranean diet. Eur J Clin Nutr 2004;58:1550-2. 